\title{
Lettre à la rédaction : \\ HAIRVEQ 2005 : contrôle externe de quali- té pour le dosage des stupéfiants dans les cheveux, réalisé en collaboration avec la Society of Hair Testing
}

\section{Letter to the editor: \\ HAIRVEQ 2005: An external quality control exercise for drugs of abuse analysis in hair in cooperation with Society of Hair Testing}

Simona PICHINI*(1), Montserrat VENTURA ${ }^{(2,3)}$, Mitona PUJADAS ${ }^{(2)}$, Rosa VENTURA ${ }^{(2,4)}$, Rita DI GIOVANNANDREA ${ }^{(1)}$, Piergiorgio ZUCCARO ${ }^{(1)}$, Roberta PACIFICI ${ }^{(1)}$, Rafael De La TORRE ${ }^{(2,4)}$, Carmen JURADO ${ }^{(5)}$

(1) Drug Control and Evaluation Department, Istituto Superiore di Sanità - Rome - Italy (2) Unitat de Recerca en Farmacologia, Institut Municipal d'Investigaciò Médica - Barcelona - Spain (3) UDIMAS, Universitat Autonoma de Barcelona - Barcelona - Spain (4) CEXS, Universitat Pompeu Fabra - Barcelona - Spain

(5) Instituto Nacional de Toxicologia y Ciencias Forenses, Departamento de Sevilla - Spain

*Corresponding Author: Simona PICHINI, Drug Control and Evaluation Department, Istituto Superiore di Sanità, V. le Regina Elena 299, 00161 Rome - Italy Tel : +3906 49903682 - Fax : +390649902016 - E-mail : pichini@iss.it 


\section{Introduction}

Since 2002 the Istituto Superiore di Sanità of Rome, Italy, in cooperation with Institut Municipal d'Investigaciò Mèdica of Barcelona, Spain, has set up an external quality control program (HAIRVEQ) to evaluate reliability in hair testing for drug abuse by laboratories from the Italian National Health Service (1). In the year 2005, three samples included in the first round were also included in the set of samples of the proficiency test (PT) organized by the Society of Hair Testing (SoHT) in a joint exercise (2). The aim was to compare methodological strategies, qualitative and quantitative results obtained by two different groups of Laboratories: those from the Italian National Health Service, which are not specifically devoted to hair analysis and those from the Society of Hair Testing, generally skilled for keratin matrix assays.

\section{Methods}

Actual participants in HAIRVEQ are thirty-two laboratories operating within the Italian National Health service and two foreign laboratories of pharmacotoxicology. Twenty-six laboratories reported results for this exercise (HAIRVEQ Laboratories). Twenty-one laboratories of pharmacotoxicology from different European and non European countries were registered to participate in the PT of SoHT and seventeen of them sent the results on time for this exercise (SoHT Laboratories).

Four reference Laboratories evaluated qualitative and quantitative content of hair samples and values were reported as mean \pm standard deviation (SD) (Table 1). The three samples included in both HAIRVEQ 2005 and the proficiency test of SoHT 2005 were the following: sample A: a blank control sample; sample B: containing 3,4-methylenedioxymethamphetamine (MDMA) and 3,4-methylenedioxyamphetamine (MDA); sample C: containing cocaine, benzoylecgonine (BEG) $\Delta^{9}$-tetrahydrocannabinol (THC) and 11-nor$\Delta^{9}$-tetrahydrocannabinol-9-carboxylic acid (THC$\mathrm{COOH}$ ). In this latter case, statistical evaluation was not possible since only four participating laboratories from both groups evaluated this analyte.

Results obtained by participating laboratories were classified according to $\mathrm{Z}$-score value (calculated as ( $\mathrm{x}$ $\mathrm{X}) / \mathrm{s}$ where $\mathrm{x}=$ value for each participating laboratory, $\mathrm{X}=$ mean of reference value, $\mathrm{s}=$ standard deviation from reference Laboratories). Results were categorized as satisfying when $|z| \leq 2$, uncertain when $2<|z|<3$, and as unsatisfying when $|z| \geq 3$.

\section{Results and discussion}

A first noticeable difference in qualitative evaluation of hair samples between laboratories participating in both HAIRVEQ and those from the SoHT, (which was encountered also in case of other the other samples), is the number of false positive results. No false positive results were reported by SoHT laboratories for the blank sample A while HAIRVEQ Laboratories gave a total of ten false positive results for opiates, cocaine and methadone.

A relatively low coefficient of variation was obtained when considering the results from the Reference Laboratories for the quantification of MDMA and MDA in sample $B$, which influenced the subsequent calculation of $Z$ score for the participating Laboratories (Table 1). It is worth noticing that Reference Laboratories did not follow a common standardized analysis method, but applied different digestion and extraction procedures. A rate of $25.0 \%$ and a $46.2 \%$ unsatisfying results were obtained for MDMA and $40 \%$ and $0 \%$ unsatisfying results were obtained for MDA by HAIRVEQ and SoHT Laboratories, respectively. Also in the case of sample $B$, a quite high number of false positive results $(n=10)$ were given by Laboratories of HAIRVEQ while fewer were given $(n=4)$ by Laboratories of SoHT.

Once again, a relatively low coefficient of variation was obtained when considering the results from the Reference Laboratories for the quantification of cocaine and BEG in sample C (Table 1). Although percentage of unsatisfying results was similar for the two groups of participating Laboratories, $Z$ scores from HAIRVEQ laboratories were always more than twice those for SoHT scores. Since in case of THC only one result was available from Reference Laboratories, $\mathrm{Z}$ score was calculated with respect to the mean and SD values obtained by the participating Labs. Obviously, better results were obtained for both HAIRVEQ and SoHT Laboratories (Table 1).

The problem of false positive results reported by HAIRVEQ Laboratories was confirmed for sample C. A total of eight false positive results for various drugs of abuse were obtained vs none from SoHT Laboratories.

No substantial differences were found when comparing methodologies applied by HAIRVEQ and SoHT Laboratories (data not shown). Nonetheless, regarding the analytical instrumentation, HAIRVEQ Labs still use techniques such as high performance liquid chromatography with ultraviolet or electrochemical detection, including the REMEDI system, which are not suited for hair testing. 
In conclusion, participating Laboratories from the SoHT showed an excellent performance in the qualitative evaluation of hair samples. Quantitative evaluation was good, even if scattered values were obtained, in agreement with results obtained in previous proficiency testing exercises $(3,4)$. Concerning laboratories participating in the HAIRVEQ exercise, few laboratories showed a satisfying performance for both qualitative and quantitative hair testing analysis. Up to now, a unique source of error has not been identified: they depend neither on the experience of the participating labs, nor by the analytical instrumentation or analytical method used. The most likely source of error resides in method validation: correct use of internal standard (several laboratories do not use any internal standard); correct method validation (some laboratories declare that they do not perform an extensive method validation); use of calibration curve for calculation (many laboratories do not give information on performing calibration curve or using blank hair samples for calibration curves) and finally use of correct cut-off (different cut-offs are used and not always matching those proposed by the SoHT (4). Next rounds of the HAIRVEQ program will contain a specific inquiry on these issues.

\section{References}

1. S. Pichini, M. Ventura, M. Pujadas, R. Ventura, M. Pellegrini, P. Zuccaro, R. Pacifici, and R. de la Torre. HAIRVEQ: an external quality control scheme for drugs of abuse analysis in hair. Forensic Sci Int. 145: 109-115 (2004).

2. C. Jurado, and H. Sachs. Proficiency test for the analysis of hair for drugs of abuse, organized by the Society of Hair Testing. Forensic Sci Int. 133: 175-178 (2003).

3. H. Sachs. Quality control by the Society of Hair Testing. Forensic Sc. Int. 84: 145-150 (1997).

4. Society of Hair Testing. Recommendations for hair testing in forensic cases. Forensic Sci Int. 145: 83-84 (2004).

Table I : Results from the joint intercomparison exercise run by HAIRVEQ and SoHT Laboratories on hair testing for drugs of abuse (samples $B$ and $C$ ).

\begin{tabular}{|c|c|c|c|c|c|c|c|c|c|c|c|}
\hline \multirow{2}{*}{$\begin{array}{l}\text { Sample } \\
\text { content }\end{array}$} & \multicolumn{3}{|c|}{ Reference Laboratories Evaluation } & \multicolumn{4}{|c|}{ HAIRVEQ I aboratories Evaluation } & \multicolumn{4}{|c|}{ SoHT Laboratories Evaluation } \\
\hline & Mean & SD & $\mathrm{CV} \%$ & Mean & SD & $\mathrm{CV} \%$ & $\begin{array}{c}\text { Unsatisfying } \\
\text { Results \% }\end{array}$ & Mean & SD & $\mathrm{CV} \%$ & $\begin{array}{l}\text { Unsatisfying } \\
\text { Results } \%\end{array}$ \\
\hline \multicolumn{12}{|l|}{ Sample B } \\
\hline MDMA & 2.5 & 0.2 & 9.5 & 2.5 & 0.9 & 34.6 & 25.0 & 2.2 & 0.9 & 42.9 & 46.2 \\
\hline $\mathrm{MDA}$ & 0.2 & 0.08 & 35.9 & 0.9 & 1.2 & 134.2 & 40.0 & 0.2 & 0.1 & 61.6 & 0.0 \\
\hline \multicolumn{12}{|l|}{ Sample C } \\
\hline Cocaine & 10.0 & 0.53 & 5.3 & 16.9 & 29.3 & 173.2 & 70.6 & 3.7 & 4.0 & 48.0 & 86.7 \\
\hline BEG & 2.7 & 0.29 & 10.9 & 10.1 & 4.8 & 107.5 & 47.1 & 3.1 & 1.6 & 53.0 & 50.0 \\
\hline THC & $3.4^{*}$ & & & 2.5 & 1.9 & 76.2 & 0.0 & 2.8 & 2.0 & 71.2 & 0 \\
\hline
\end{tabular}

*Results given by only one Reference Laboratory 